\title{
QUASICIRCLES AND THE CONFORMAL GROUP
}

\author{
YVES BENOIST AND DOMINIQUE HULIN
}

ABstract. We prove that a Jordan curve in the 2-sphere is a quasicircle if and only if the closure of its orbit under the action of the conformal group contains only points and Jordan curves.

\section{INTRODUCTION}

The 2-sphere $\mathbb{S}^{2}$, oriented and equipped with its standard conformal structure, is isomorphic to the complex projective line $\mathrm{P}^{1} \mathbb{C} \simeq \mathbb{C} \cup\{\infty\}$.

Let $K \geq 1$. By definition (see [16] or Section 2.1), a $K$-quasicircle $c \subset \mathbb{S}^{2}$ is the image $c=f\left(c_{0}\right)$ of a circle $c_{0} \subset \mathbb{S}^{2}$ under a $K$-quasiconformal homeomorphism $f: \mathbb{S}^{2} \rightarrow \mathbb{S}^{2}$.

Our aim in this paper is to characterize those Jordan curves in $\mathbb{S}^{2}$ that are quasicircles in terms of their orbit under the action of the conformal group $G:=$ $\operatorname{Conf}^{+}\left(\mathbb{S}^{2}\right) \simeq \mathrm{PSl}_{2} \mathbb{C}$.

Let $\mathcal{K}$ denote the set of nonempty compact subsets of $\mathbb{S}^{2}$ equipped with the Hausdorff distance. This space $\mathcal{K}$ is a compact metric space. Observe that, when $C \subsetneq \mathbb{S}^{2}$ is any proper compact subset of $\mathbb{S}^{2}$, the closure $\overline{G C} \subset \mathcal{K}$ of its orbit in $\mathcal{K}$ contains all singletons in $\mathbb{S}^{2}$. We thus also introduce $\mathcal{K}_{0} \subset \mathcal{K}$, the set of compact subsets of $\mathbb{S}^{2}$ distinct from a singleton.

Theorem 1.1. Let $K \geq 1$. The set of all $K$-quasicircles of $\mathbb{S}^{2}$ is a closed $G$ invariant subset of $\mathcal{K}_{0}$.

Conversely, any closed $G$-invariant subset of $\mathcal{K}_{0}$ which consists only of Jordan curves is included in the set of $K$-quasicircles for some $K \geq 11$

A straightforward consequence is the following topological characterization of quasicircles.

Corollary 1.2. A Jordan curve $c \subset \mathbb{S}^{2}$ is a quasicircle if and only if its orbit closure $\overline{G c}$ in $\mathcal{K}$ consists only of points and Jordan curves.

In other words, quasicircles are characterized among Jordan curves by the fact that "when zooming in, one sees nothing but Jordan curves".

Since they were first introduced by Pfluger and Tienari in the early 1960s, a number of various characterizations of quasicircles has been progressively discovered. This history leading to an impressive list of equivalent definitions is the subject of

Received by the editors November 4, 2016.

2010 Mathematics Subject Classification. Primary 30C62; Secondary 57M60.

Key words and phrases. Quasidisks, quasiconformal maps, Jordan curves.

${ }^{1}$ After publication of this preprint, we learned that its main result was already included in the nice paper by V. Aseev and D. Kuzin, Continua of bounded turning: chain conditions and infinitesimal connectedness, Siberian Math. J. 41 (2000), 801-810. 
the nice and recent book "The ubiquitous quasidisk" by Gehring and Hag [9]. Our new characterization has yet a different flavour.

The paper is organized as follows. In Section 2, we briefly recall the definition of quasiconformal maps and quasicircles, and prove that the limit in $\mathcal{K}$ of a convergent sequence of $K$-quasicircles is either a point or a $K$-quasicircle. This result, which is the first part of Theorem 1.1. follows readily from a standard compactness result for $K$-quasiconformal homeomorphisms of $\mathbb{S}^{2}$. We also recall the so-called Ahlfors' arc condition, which is a criterion for a Jordan curve in $\mathbb{S}^{2}$ to be a quasicircle. In Section 3 we outline the proof of the second part of Theorem 1.1. It involves three intermediate results: Propositions [3.1, 3.3, and 3.4. We fill in the details of these three propositions in Section 4 where we address topology of the plane, in Section 5 where we consider maximal disks in Jordan domains of $\mathbb{S}^{2}$, and in Section 6 where we examine finite sequences of real numbers. We wrap up the proof of Theorem 1.1 in Section 7 .

In Section 8, we will explain an analog of Theorem 1.1 where Jordan curves are replaced by Cantor sets (Theorem 8.1). In Section 9, we give an elementary proof of a technical result (Proposition 5.6) which is needed in our proof.

\section{LiMITS OF $K$-QUASICIRCLES}

In this section, we prove the first part of Theorem 1.1. It relies on the classical compactness property of $K$-quasiconformal maps (Theorem 2.11). We also recall Ahlfors' characterization of quasicircles (Theorem 2.4).

2.1. Quasiconformal maps and quasicircles. Let us first recall the definition of quasiconformal maps (see [2] or [13]).

A quadrilateral $Q$ is a Jordan domain in $\mathbb{S}^{2}$, together with a cyclically ordered quadruple of boundary points. We say that two quadrilaterals $Q$ and $Q^{\prime}$ are conformally equivalent when there exists a homeomorphism $\varphi: \bar{Q} \rightarrow \overline{Q^{\prime}}$ between their closures that sends the vertices of $Q$ to the vertices of $Q^{\prime}$, and whose restriction $\varphi: Q \rightarrow Q^{\prime}$ is a conformal map.

Any quadrilateral $Q$ is conformally equivalent to a rectangle $R$ with vertices $(0, x, x+i y, i y)$ where $x$ and $y$ are positive. The conformal modulus of the quadrilateral $Q$ is then defined as $m(Q)=m(R):=x / y$.

A homeomorphism $f: \mathbb{S}^{2} \rightarrow \mathbb{S}^{2}$ is said to be $K$-quasiconformal $(K \geq 1)$ if the inequalities

$$
K^{-1} m(Q) \leq m(f(Q)) \leq K m(Q)
$$

hold for any quadrilateral $Q \subset \mathbb{S}^{2}$.

A conformal homeomorphism $f: \mathbb{S}^{2} \rightarrow \mathbb{S}^{2}$ is 1-quasiconformal. It can be proved that the converse is true, that is, a 1-quasiconformal homeomorphism is actually conformal [13, Theorem I.5.1]. It follows immediately from the definition that, when $f_{i}: \mathbb{S}^{2} \rightarrow \mathbb{S}^{2}$ are $K_{i}$-quasiconformal homeomorphisms $(i=1,2)$, the composed map $f_{1} \circ f_{2}: \mathbb{S}^{2} \rightarrow \mathbb{S}^{2}$ is $K_{1} K_{2}$-quasiconformal.

As already mentioned, a $K$-quasicircle $c \subset \mathbb{S}^{2}$ is the image $c=f\left(c_{0}\right)$ of a circle $c_{0} \subset \mathbb{S}^{2}$ under a $K$-quasiconformal homeomorphism $f: \mathbb{S}^{2} \rightarrow \mathbb{S}^{2}$.

2.2. Compactness for quasiconformal maps. Equip the 2-sphere $\mathbb{S}^{2}$ with its canonical Riemannian metric $d$. The main property of quasiconformal maps that will be used in this paper is the following fundamental compactness theorem. 
Theorem 2.1 ([13, Theorems II.5.1 and II.5.3]). Let $K \geq 1$, let $z_{1}, z_{2}, z_{3}$ be three distinct points in $\mathbb{S}^{2}$, and let $f_{n}: \mathbb{S}^{2} \rightarrow \mathbb{S}^{2}$ be a sequence of $K$-quasiconformal homeomorphisms such that the three sequences $\left(f_{n}\left(z_{i}\right)\right)_{n \geq 1}$ converge to three distinct points. Then, there exists a subsequence $\left(f_{n_{k}}\right)$ and a K-quasiconformal homeomorphism $f: \mathbb{S}^{2} \rightarrow \mathbb{S}^{2}$ such that $f_{n_{k}} \rightarrow f$ uniformly on $\mathbb{S}^{2}$.

We will infer that the limit of a sequence of $K$-quasicircles that converges to a compact set which is not a point is also a $K$-quasicircle. Moreover, we will prove that one can choose parameterizations for this sequence of quasicircles that converge to a parameterization of the limit. This means that a convergent sequence of $K$-quasicircles cannot fold several times over its limit, that is, the configuration in Figure 1 is forbidden.

Let $\mathbb{S}^{1}:=\mathbb{R} \cup\{\infty\}$ denote the standard circle in $\mathbb{S}^{2} \simeq \mathbb{C} \cup\{\infty\}$.

Proposition 2.2. Let $c_{n} \subset \mathbb{S}^{2}$ be a sequence of $K$-quasicircles that converges in $\mathcal{K}$ to a compact set $c_{\infty} \subset \mathbb{S}^{2}$ which is not a point. After going to a subsequence if necessary, there exist $K$-quasiconformal homeomorphisms $f_{n}: \mathbb{S}^{2} \rightarrow \mathbb{S}^{2}$ and $f_{\infty}: \mathbb{S}^{2} \rightarrow \mathbb{S}^{2}$ with $c_{n}=f_{n}\left(\mathbb{S}^{1}\right), c_{\infty}=f_{\infty}\left(\mathbb{S}^{1}\right)$, and such that $f_{n} \rightarrow f_{\infty}$ uniformly on $\mathbb{S}^{2}$.

Proof. Let $h_{n}$ be a $K$-quasiconformal homeomorphism of $\mathbb{S}^{2}$ such that $c_{n}=h_{n}\left(\mathbb{S}^{1}\right)$. The limit $c_{\infty}=\lim c_{n}$, as a limit of compact connected sets, is also compact and connected. Since $c_{\infty}$ is not a singleton, it contains at least two and hence three distinct points $x_{\infty}, y_{\infty}$ and $z_{\infty}$.

For each $n \in \mathbb{N}$, one can pick three distinct points $x_{n}, y_{n}, z_{n}$ in $\mathbb{S}^{1}$ such that $h_{n}\left(x_{n}\right) \rightarrow x_{\infty}, h_{n}\left(y_{n}\right) \rightarrow y_{\infty}$ and $h_{n}\left(z_{n}\right) \rightarrow z_{\infty}$. Let $\gamma_{n} \in \mathrm{PSl}_{2} \mathbb{R} \subset \mathrm{PSl}_{2} \mathbb{C}$ be the conformal transformation of $\mathbb{S}^{2}$ that preserves $\mathbb{S}^{1}$ and that sends 0 to $x_{n}, 1$ to $y_{n}$ and $\infty$ to $z_{n}$. Each map $f_{n}:=h_{n} \circ \gamma_{n}: \mathbb{S}^{2} \rightarrow \mathbb{S}^{2}$ is still a $K$-quasiconformal homeomorphism of $\mathbb{S}^{2}$ such that $c_{n}=f_{n}\left(\mathbb{S}^{1}\right)$. We now have $f_{n}(0) \rightarrow x_{\infty}, f_{n}(1) \rightarrow$ $y_{\infty}$ and $f_{n}(\infty) \rightarrow z_{\infty}$. Thus Theorem 2.1 applies to the sequence $\left(f_{n}\right)$ and yields the result.

This proves the first part of Theorem 1.1, To prove the second part of Theorem 1.1. we will use the following characterization of quasicircles due to Ahlfors.

2.3. Ahlfors' arc condition. We recall that a Jordan arc $a \subset \mathbb{S}^{2}$ is a subset of $\mathbb{S}^{2}$ which is homeomorphic to the closed interval $[0,1]$.

Definition 2.3. A Jordan curve $c \subset \mathbb{S}^{2}$ satisfies the arc condition with constant $A \geq 1$ if, for any pair of points $x, z \in c$ delimiting two Jordan $\operatorname{arcs} a_{+}, a_{-}$on $c$, their diameters satisfy

$$
\min \left(\operatorname{diam}\left(a_{+}\right), \operatorname{diam}\left(a_{-}\right)\right) \leq A d(x, z) .
$$

Theorem 2.4 (Ahlfors; see [1] and [9, Theorem 2.2.5]). A Jordan curve $c \subset \mathbb{S}^{2}$ is a quasicircle if and only if it satisfies the arc condition.

The implied constants depend only on each other.

\section{An OVERVIEW of the Proof of TheOrem 1.1}

We sketch the proof of the second part of Theorem 1.1 It will consist of four propositions that will be proved in the following sections. 
We assume that

$\mathcal{F}$ is a closed $G$-invariant subset of $\mathcal{K}_{0}$, which consists only of

Jordan curves and such that, for all $A \geq 1$, there exists a Jordan curve in $\mathcal{F}$ that does not satisfy Ahlfors' arc condition with constant $A$.

Under this assumption, we want to find a contradiction. The first step is the following and will be completed in Section 4

Proposition 3.1 (Two threads with the same limit). Assume (3.1). Then, there exist a sequence $\left(c_{n}\right)$ in $\mathcal{F}$ that converges to a Jordan curve $c_{\infty} \in \mathcal{F}$, a Jordan arc $a_{\infty} \subset c_{\infty}$ and, for each $n \in \mathbb{N}$, two disjoint Jordan arcs $a_{n}$ and $a_{n}^{\prime}$ in $c_{n}$ such that both sequences $\left(a_{n}\right)$ and $\left(a_{n}^{\prime}\right)$ converge to $a_{\infty}$ when $n \rightarrow \infty$.

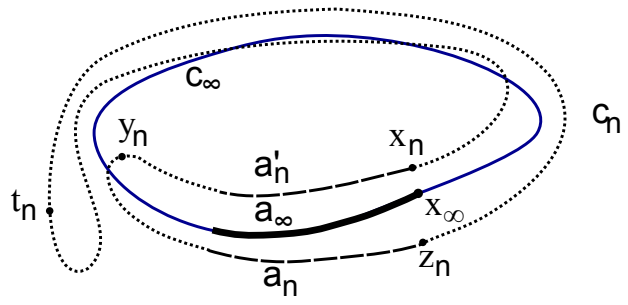

Figure 1. Two threads with the same limit. Note that this cannot happen in a convergent sequence of $K$-quasicircles.

We will assume from now on, without loss of generality, that the Jordan $\operatorname{arc} a_{\infty}$ lies in $\mathbb{C} \subset \mathbb{C} \cup\{\infty\} \sim \mathbb{S}^{2}$. What we have in mind to obtain our contradiction is now to zoom in, that is, to replace each $c_{n}$ by another Jordan curve $\gamma_{n} c_{n} \in \mathcal{F}$ where $\gamma_{n} \in \mathrm{PSl}_{2} \mathbb{C}$ fixes the point $\infty$, and examine the behaviour of the arcs $\gamma_{n} a_{n}$ and $\gamma_{n} a_{n}^{\prime}$.

To achieve this goal, we will first associate to each one of the Jordan curves $c_{n}$ a "pearl necklace", that is, a sequence of disks roughly joining the endpoints of $a_{\infty}$ and channelled by the $\operatorname{arcs} a_{n}$ and $a_{n}^{\prime}$ (see Figure 2). This necklace will grow thinner as $n \rightarrow \infty$. The precise statement, which constitutes our second step and will be proved in Section [5, is as follows.

Definition 3.2. Let $U \subsetneq \mathbb{C}$ be an open subset. A necklace $N=(D(i) \mid i \in I)$ in $U$ is a sequence of open disks $D(i) \subset U$ of the complex plane, indexed by a finite interval $I \subset \mathbb{Z}$, and that satisfy the following conditions:

(1) two consecutive disks $D(i)$ and $D(i+1)$ intersect orthogonally,

(2) when $|i-j| \geq 2$, the disks $D(i)$ and $D(j)$ do not intersect,

(3) for each three consecutive disks, the set $\partial D(i) \backslash(D(i-1) \cup D(i+1))$ is a disjoint union of two arcs that both meet the boundary $\partial U$.

The thickness of the necklace $N$ is the ratio

$$
\max _{i \in I} \operatorname{diam} D(i) / \operatorname{diam}\left(\bigcup_{i \in I} D(i)\right)
$$

where diam denotes the diameter with respect to the Euclidean distance on $\mathbb{C}$. The necklace is said to be $\varepsilon$-thin if its thickness is bounded by $\varepsilon$. 


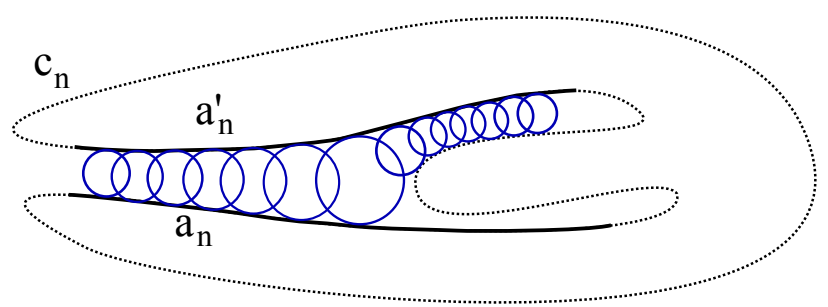

Figure 2. A pearl necklace.

Proposition 3.3 (Thin pearl necklaces). Assume (3.1). Then, for each $\varepsilon>0$, there exist a Jordan curve $c_{\varepsilon} \in \mathcal{F}$ and an $\varepsilon$-thin necklace $N_{\varepsilon}=\left(D_{\varepsilon}(i) \mid i \in I_{\varepsilon}\right)$ in the domain $U_{\varepsilon}=\mathbb{C} \backslash c_{\varepsilon}$.

As mentioned above, the last step of the proof will consist in zooming in on a well-chosen pearl of each of these necklaces to obtain a contradiction. To this effect, we associate to each necklace $N_{\varepsilon}$ provided by Proposition 3.3 the sequence

$$
x_{\varepsilon}=\left(x_{\varepsilon}(i) \mid i \in I_{\varepsilon}\right), \text { where } x_{\varepsilon}(i)=\log \operatorname{diam} D_{\varepsilon}(i),
$$

of the logarithms of the diameters of the $D_{\varepsilon}(i)$ 's.

Our third proposition is a very general statement on families of sequences of real numbers and will be proved in Section 6 .

Proposition 3.4 (Finite sequences of real numbers). Let $\mathcal{S}$ be a family of sequences $x=\left(x(i) \mid i \in I_{x}\right)$ of real numbers, indexed by finite intervals $I_{x} \subset \mathbb{Z}$. Then at least one of the following three possibilities occurs:

(1) There are pipes in $\mathcal{S}$ : there exists a thickness $h_{1}>0$ such that, for any length $\ell$, there exist a sequence $x \in \mathcal{S}$ and a subinterval $J \subset I_{x}$ with length $|J|=\ell$ such that

$$
|x(i)-x(j)| \leq h_{1} \quad \text { for any } i, j \in J .
$$

(2) There are wells in $\mathcal{S}$ : for any depth $h_{2}>0$, there exist a sequence $x \in \mathcal{S}$ and $i<j<k$ in $I_{x}$ such that

$$
x(j)<x(i)-h_{2} \text { and } x(j)<x(k)-h_{2} .
$$

(3) All sequences in $\mathcal{S}$ are slides: there exist a height $h_{3} \in \mathbb{R}$ and a slope $\sigma>0$ such that for any sequence $x \in \mathcal{S}$ and any index $i_{0} \in I_{x}$ such that $x\left(i_{0}\right)=\max \left\{x(i), i \in I_{x}\right\}$, one has

$$
x(i) \leq x\left(i_{0}\right)+h_{3}-\sigma\left|i-i_{0}\right| \text { for any } i \in I_{x} .
$$

By applying Proposition 3.4 to the family $\mathcal{S}_{\mathcal{F}}$ of finite sequences associated to the necklaces in Proposition 3.3 we will obtain Proposition 3.5 which provides us with the desired contradiction.

Proposition 3.5 (Excluding the three cases). Assume (3.1). Then, there are no pipes in $\mathcal{S}_{\mathcal{F}}$. There are no wells in $\mathcal{S}_{\mathcal{F}}$. Not all the sequences in $\mathcal{S}_{\mathcal{F}}$ are slides.

Proposition 3.5 will be proved in Section 7 . 


\section{Constructing the two threads}

Proof of Proposition 3.1. Recall that $\mathcal{F} \subset \mathcal{K}_{0}$ is a closed $G$-invariant subset of $\mathcal{K}_{0}$ which consists only of Jordan curves, and that the Jordan curves in $\mathcal{F}$ do not satisfy a uniform Ahlfors' arc condition. This means that we can find a sequence $c_{n} \in \mathcal{F}$ and, for each $n \in \mathbb{N}$, a cyclically ordered quadruple $\left(x_{n}, y_{n}, z_{n}, t_{n}\right)$ of points on $c_{n}$ such that

$$
\min \left(d\left(x_{n}, y_{n}\right), d\left(x_{n}, t_{n}\right)\right) \geq n d\left(x_{n}, z_{n}\right) .
$$

Note that this forces $d\left(x_{n}, z_{n}\right) \rightarrow 0$. Replacing if necessary each Jordan curve $c_{n}$ by $\gamma_{n}\left(c_{n}\right) \in \mathcal{F}$, where $\gamma_{n} \in \mathrm{PSl}_{2} \mathbb{C}$ is a suitable zoom in on the point $x_{n}$, we may assume moreover that

$$
r:=\inf _{n \in \mathbb{N}} \min \left(d\left(x_{n}, y_{n}\right), d\left(x_{n}, t_{n}\right)\right) \text { is positive. }
$$

We want to prove that there exist, for each $n \in \mathbb{N}$, two disjoint Jordan $\operatorname{arcs} a_{n}$ and $a_{n}^{\prime}$ in $c_{n}$ that converge to the same Jordan $\operatorname{arc} a_{\infty}$ when $n \rightarrow \infty$.

The sphere $\mathbb{S}^{2}$ and the space $\mathcal{K}$ are compact metric spaces. Going to a subsequence, we may thus also assume that:

- there exist three points $x_{\infty}, y_{\infty}$ and $t_{\infty}$ in $\mathbb{S}^{2}$ with $x_{\infty} \neq y_{\infty}$ and $x_{\infty} \neq t_{\infty}$, and such that

$$
x_{n} \rightarrow x_{\infty}, z_{n} \rightarrow x_{\infty}, y_{n} \rightarrow y_{\infty}, t_{n} \rightarrow t_{\infty} \quad \text { when } n \rightarrow \infty,
$$

- the sequence of Jordan curves $\left(c_{n}\right)$ converges in $\mathcal{K}$. Its limit $c_{\infty}$ is not a singleton since it contains the points $x_{\infty} \neq y_{\infty}$. Since $\mathcal{F}$ is a closed subset of $\mathcal{K}_{0}$, the limit $c_{\infty}$ belongs to $\mathcal{F}$ : it is a Jordan curve.

Consider the four Jordan arcs

$$
\left[x_{n}, y_{n}\right],\left[x_{n}, t_{n}\right],\left[z_{n}, y_{n}\right] \text { and }\left[z_{n}, t_{n}\right] \text {. }
$$

We shorten each of them, keeping the first endpoint $x_{n}$ or $z_{n}$, in order to get a Jordan arc whose diameter is exactly $r / 2$. Going again to a subsequence, we may assume that each one of these four sequences of shortened arcs converges in $\mathcal{K}$. Their respective limits $\alpha_{1}, \alpha_{2}, \alpha_{3}$ and $\alpha_{4}$ are compact connected subsets of $c_{\infty}$ that contain $x_{\infty}$ and have diameter $r / 2$ so that they all are Jordan $\operatorname{arcs}$ in $c_{\infty}$. Thus there exists a Jordan arc $a_{\infty} \subset c_{\infty}$ that contains $x_{\infty}$ as an endpoint and that is a subarc of at least two of the limit sets $\alpha_{i}$ (see Figure 11). Proposition [3.1] now follows from Lemma 4.1 .

Lemma 4.1. Let $\alpha_{n} \subset \mathbb{S}^{2}$ be a sequence of Jordan arcs converging to a Jordan arc $\alpha_{\infty}$. Let $a_{\infty}$ be a Jordan subarc of $\alpha_{\infty}$. Then, there exists a sequence of Jordan subarcs $a_{n} \subset \alpha_{n}$ such that $a_{n}$ converges to $a_{\infty}$.

Proof. Using Jordan's theorem, one may assume that $\alpha_{\infty} \subset \mathbb{S}^{1}$ and $a_{\infty}=[-1,1]$. The proof in this case is left to the reader.

\section{Pearl necklaces}

5.1. Normal disks and Thurston's stratification. Recall that $\mathbb{S}^{2}$ is equipped with its canonical Möbius structure. Let $U \subset \mathbb{S}^{2}$ be a connected domain that avoids at least two points. W. Thurston introduced a stratification of the domain $U$ associated to the family of maximal disks $D \subset U$ sitting in $U$. We briefly recall the construction of this stratification, and the facts we will be using in this paper. 
Any disk $D \subset \mathbb{S}^{2}$ carries a conformal hyperbolic metric, whose geodesics are arcs of circles that cut the boundary $\partial D$ of $D$ orthogonally. The convex hull, for this metric, of a subset $A \subset \partial D$ will be denoted by $\operatorname{conv}_{D}(A) \subset D$.

Definition 5.1. An open disk $D \subset U$ is normal when its boundary $\partial D$ meets $\partial U$ in at least two points. When $D \subset U$ is a normal disk, define

$$
C(D)=\operatorname{conv}_{D}(\partial D \cap \partial U) .
$$

Note that a normal disk $D \subset U$ is maximal among the disks sitting in $U$.

Proposition 5.2 (W. Thurston). For any point $p \in U$, there exists a unique normal disk $D_{p} \subset U$ such that $p \in C\left(D_{p}\right)$. This disk $D_{p}$ depends continuously on the point $p \in U$.

This means that the convex hulls $C(D)$ of all the normal disks provide a stratification of $U$.

A proof of this proposition is given in [11, Theorem 1.2.7], [12, 7] or [3, Chapter 4]. See also [15] and [8] for other applications of this construction. For the convenience of the reader we include a short and elementary proof.

Proof of Proposition 5.2. We may assume $U \subset \mathbb{C}$.

Uniqueness. Just notice that, for any two open disks $D_{1}$ and $D_{2}$ in $\mathbb{C}$, the convex hulls $\operatorname{Conv}_{D_{1}}\left(\partial D_{1} \backslash D_{2}\right)$ and $\operatorname{Conv}_{D_{2}}\left(\partial D_{2} \backslash D_{1}\right)$ do not meet.

Existence. Let $p \in U$. We will use the inversion $j_{p}: z \mapsto(z-p)^{-1}$ of the sphere. We introduce the compact subset $K_{p}:=j_{p}\left(\mathbb{S}^{2} \backslash U\right)$ of $\mathbb{C}$. Recall that there exists a unique closed disk $\Delta_{p}$ of $\mathbb{C}$ with minimal radius that contains $K_{p}$. Moreover the intersection $\partial \Delta_{p} \cap \partial K_{p}$ is not included in an open arc of $\partial \Delta_{p}$ whose endpoints are diametrically opposed. Thus the open disk $D_{p}:=j_{p}^{-1}\left(\mathbb{S}^{2} \backslash \Delta_{p}\right)$ is a normal disk of $U$ and the point $p$ belongs to the convex hull $C\left(D_{p}\right)$.

Continuity. Since the compact set $K_{p}$ depends continuously on the point $p$, the disks $\Delta_{p}$ and $D_{p}$ also depend continuously on $p$.

Notation 5.3. When $p \in U$, we will denote by $C_{p}:=C\left(D_{p}\right) \subset U$ the stratum that contains the point $p$.

When $D \subset U$ is a normal disk, let $\lambda(D) \subset U$ denote the boundary in the disk $D$ of the convex hull $C(D)$. This boundary $\lambda(D)$ has a finite or countable number of connected components, which are arcs of circles.

Assume from now on the domain $U$ to be simply connected. Then, when $D \subset U$ is a normal disk, $U \backslash C(D)$ is not connected. More precisely, we have the following lemma whose proof is left to the reader.
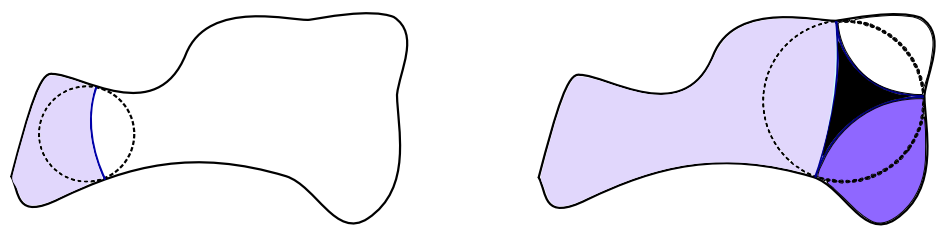

Figure 3. A normal disk $D$ and the connected components of $U \backslash C(D)$. 
Lemma 5.4. Let $U \subset \mathbb{S}^{2}$ be a simply connected domain that avoids at least two points, and let $D \subset U$ be a normal disk. If $\partial D \cap \partial U$ contains only two points, then $U \backslash C(D)$ has two connected components. If $\partial D \cap \partial U$ contains at least three points, then there is a natural bijection between the set of connected components $\Omega$ of $U \backslash C(D)$ and the set of connected components of $\lambda(D)$. It is given by $\Omega \longrightarrow \partial \Omega \cap U$.

5.2. Monotonous paths. We now introduce monotonous paths in the simply connected domain $U$.

Definition 5.5. Let $x, y, z$ be three points of $U$. We say that $y$ lies between $x$ and $z$ if one cannot find a connected component of $U \backslash C_{y}$ that contains both $x$ and $z$. A path $\gamma:[0,1] \rightarrow U$ is monotonous if, for any parameters $0 \leq r \leq s \leq t \leq 1$, the point $\gamma(s)$ lies between $\gamma(r)$ and $\gamma(t)$.
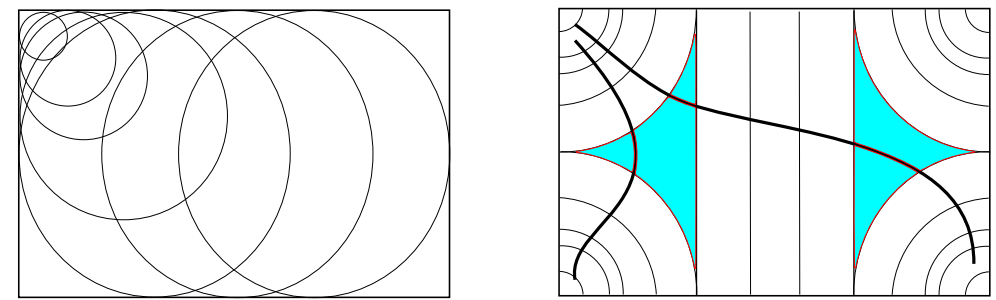

Figure 4. Maximal disks, the stratification and monotonous paths in a rectangle.

Proposition 5.6 (Monotonous paths). Let $U \subset \mathbb{S}^{2}$ be a simply connected domain that avoids at least two points. Let $p, q \in U$. Then there exists a monotonous path $\mu:[0,1] \rightarrow U$ with $\mu(0)=p$ and $\mu(1)=q$.

A proof of this proposition is given in [12, Section 11.1], where $\mu$ is obtained as a geodesic for the Thurston metric. Recall that the Thurston metric is a complete $C^{1,1}$ metric on $U$ with nonpositive curvature, for which the strata $C_{p}(p \in U)$ are convex (see [12, Section 5] or [5]; see also [10, Chapter 5] for more general constructions of monotonous paths).

We will give an elementary proof of Proposition 5.6 in Section 9.

5.3. Pearl necklaces. To a monotonous path between $p, q \in U$, we will associate a pearl necklace.

Proposition 5.7 (Pearl necklaces). Let $U \subset \mathbb{S}^{2}$ be a simply connected domain that avoids at least two points. Let $p, q \in U$. Then there exists a pearl necklace $(D(i))_{1 \leq i \leq n}$ in $U$ that joins the points $p$ and $q$, that is, such that $D(1)=D_{p}$ and $D(n) \cap D_{q} \neq \emptyset$.

The proof will follow from a series of lemmas that describe the behaviour of the normal disks along a monotonous path.

Notation 5.8. Let $\mu:[0,1] \rightarrow U$ be a monotonous path and $s \in[0,1]$, We let $\Omega_{s}^{-}$ (resp. $\Omega_{s}^{+}$) be the connected component of $U \backslash C_{\mu(s)}$ that meets $\mu([0, s]$ ) (resp. $\mu([s, 1]))$ if such a connected component does exist. Otherwise we let $\Omega_{s}^{-}$(resp. $\Omega_{s}^{+}$) be the empty set. 
Roughly, starting at time $s$, the past of $\mu$ lies in $\Omega_{s}^{-}$and its future lies in $\Omega_{s}^{+}$.

Lemma 5.9. When $0 \leq r \leq s \leq t \leq 1$, we have the inclusions

$$
D_{\mu(t)} \backslash D_{\mu(s)} \subset \Omega_{s}^{+} \quad \text { and } \quad D_{\mu(r)} \backslash D_{\mu(s)} \subset \Omega_{s}^{-} .
$$

Proof. It suffices to prove the first assertion. Assume that $D_{\mu(s)} \neq D_{\mu(t)}$. Then, $C_{\mu(t)}$ is connected and disjoint from $C_{\mu(s)}$. Since $\mu(t) \in C_{\mu(t)}$, it follows that $C_{\mu(t)} \subset \Omega_{s}^{+}$. The result follows since $D_{\mu(t)} \backslash D_{\mu(s)}$ is connected and $C_{\mu(t)}$ does not entirely lie in $D_{\mu(s)}$.

Lemma 5.10. Let $0 \leq r \leq s \leq t \leq 1$.

a) We have the inclusion $D_{\mu(r)} \cap D_{\mu(t)} \subset D_{\mu(s)}$.

b) If $D_{\mu(s)}$ meets both $D_{\mu(r)}$ and $D_{\mu(t)}$ and is equal to neither of them, the set $\partial D_{\mu(s)} \backslash\left(D_{\mu(r)} \cup D_{\mu(t)}\right)$ is a disjoint union of two arcs, each of them meeting the boundary $\partial U$.
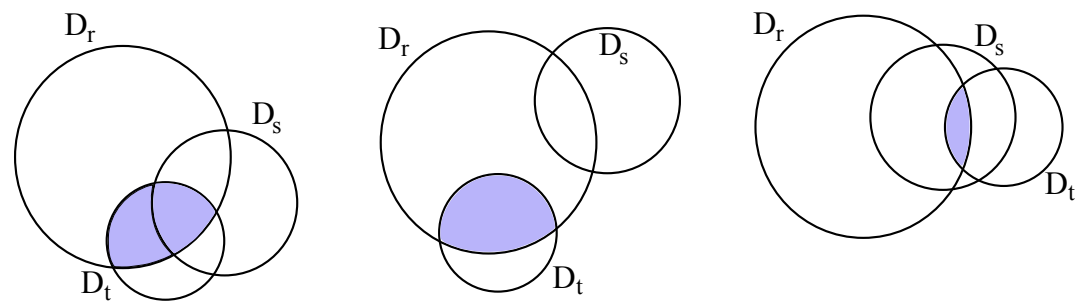

Figure 5. Two forbidden configurations, and a legit one $(r<s<t)$.

Proof. a) Lemma 5.9 ensures that $\left(D_{\mu(r)} \cap D_{\mu(t)}\right) \backslash D_{\mu(s)}$ lies in both $\Omega_{s}^{-}$and $\Omega_{s}^{+}$, hence is empty.

b) If $\partial D_{\mu(s)} \cap \partial U$ were included in only one arc of $\partial D_{\mu(s)} \backslash\left(D_{\mu(r)} \cup D_{\mu(t)}\right)$, then the points $\mu(r)$ and $\mu(t)$ would belong to the same connected component of $U \backslash C_{\mu(s)}$. A contradiction to the monotonicity of $\mu$.

Lemma 5.11. Let $r \in[0,1]$. Then, there exists at most one disk $D_{\mu(s)}$, with $s \geq r$, that is orthogonal to the disk $D_{\mu(r)}$.

Proof. Assume that $r<s<t$ and that $D_{\mu(r)}$ is orthogonal to both $D_{\mu(s)}$ and $D_{\mu(t)}$. Lemma 5.10 forces the inclusion $D_{\mu(t)} \subset D_{\mu(s)}$ of these maximal disks, hence the equality $D_{\mu(t)}=D_{\mu(s)}$.

Proof of Proposition 5.7. Let $p, q \in U$. According to Proposition 5.6, there exists a monotonous path $\mu:[0,1] \rightarrow U$ from $p$ to $q$. Let $t_{1}=0$ and define recursively, when it is possible, $t_{i+1} \in\left[t_{i}, 1\right]$ as the only parameter in the future of $t_{i}$ for which the disks $D(i):=D_{\mu\left(t_{i}\right)}$ and $D(i+1):=D_{\mu\left(t_{i+1}\right)}$ are orthogonal (Lemma 5.11).

We end up with a chain of normal disks $(D(i))$, where $i \geq 1$. Since the distance of the image $\mu([0,1]) \subset \mathbb{S}^{2}$ to ${ }^{c} U$ is nonzero, the diameters of these disks are uniformly bounded below, hence the orthogonality of consecutive disks ensures that this chain has finite cardinality $n$.

By construction, there is no parameter $t \in\left[t_{n}, 1\right]$ with $D_{\mu(t)}$ orthogonal to $D(n)$. Hence, since the normal disk $D_{x}$ depends continuously on the point $x \in U$, it follows 
that the disk $D(n)$ intersects $D_{\mu(t)}$ for every $t \in\left[t_{n}, 1\right]$. In particular, $D(n)$ meets $D_{q}$.

We must now prove that this chain is a necklace in $U$. Conditions (1) and (3) in Definition 3.2 follow from the construction and from Lemma 5.10. We now check condition (2). Let $1 \leq i<j \leq n$, with $j-i \geq 2$; we want to prove that $D(i)$ and $D(j)$ are disjoint. According to Lemma 5.10, the intersection $D(i) \cap D(j)$ is included in $D(i) \cap D(i+2)$. This is empty since both $D(i)$ and $D(i+2)$ intersect $D(i+1)$ orthogonally and since, by the same Lemma 5.10, the set $\partial D(i+1) \backslash(D(i) \cup D(i+2))$ is a union of two disjoint arcs.

\subsection{Thin necklaces.}

We prove Proposition 3.3. Recall that we assume that the arc $a_{\infty}$ provided by Proposition 3.1 lies in $\mathbb{C}$.

As mentioned in the previous section, there always exist pearl necklaces in any domain $U \subsetneq \mathbb{C}$. However the thickness of these necklaces (see Definition 3.2) may well be uniformly bounded below. It is the case for example when $U$ is a triangle. On the contrary, one can find arbitrarily thin necklaces in a domain whose boundary is a piecewise $C^{1}$ curve that admits a cusp.
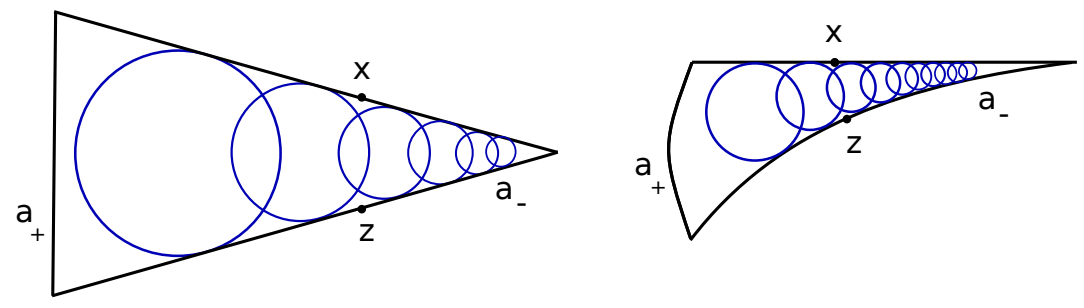

FiguRE 6. A triangle satisfies the Ahlfors' arc condition, and it does not contain arbitrarily thin necklaces. A Jordan domain with a cusp does not satisfy the Ahlfors' arc condition, and it contains arbitrarily thin necklaces.

Let $\left(c_{n}\right)$ be the sequence of Jordan curves in $\mathcal{F}$ provided by Proposition 3.1 We will use Proposition 5.7 to construct a necklace of $\mathbb{S}^{2} \backslash c_{n}$ that is drawn within a small neighbourhood of the arc $a_{\infty}$ and that roughly joins its endpoints. This necklace will grow thinner when $n \rightarrow \infty$. We begin with a general lemma.

Lemma 5.12. Let $a \subset \mathbb{C}$ be a Jordan arc. Let $\varepsilon>0$.

(1) There exists $\eta>0$ such that the diameter of any disk lying in the $\eta$ neighbourhood of a is at most $\varepsilon$.

(2) There exists $r>0$ such that, for any Jordan curve $\Gamma$ sitting in the $r$ neighbourhood of a, the bounded connected component of $\mathbb{C} \backslash \Gamma$ lies in the $\eta$-neighbourhood of the arc a.

Proof. (1) If $V_{\eta}(a)$ denotes the $\eta$-neighbourhood of $a$, one has $a=\bigcap_{\eta>0} V_{\eta}(a)$. Proceed by contradiction and assume that there exist $r_{0}>0$ and a sequence of disks $D\left(x_{n}, r_{0}\right)$ of center $x_{n} \in \mathbb{C}$ and radius $r_{0}$ that are included in $V_{1 / n} a$. The sequence $\left(x_{n}\right)_{n \geq 1}$ being bounded, we may assume that it converges to $x_{\infty}$. We would then have $D\left(x_{\infty}, r_{0}\right) \subset a$, a contradiction. 
(2) The statement is obvious when $a=[0,1]$ is a segment. Jordan's theorem yields a homeomorphism $\varphi: \mathbb{C} \rightarrow \mathbb{C}$ with $\varphi(a)=[0,1]$. The result follows, since both $\varphi$ and $\varphi^{-1}$ are locally uniformly continuous.

We may now proceed with our construction.

Proof of Proposition 3.3. Let $\left(c_{n}\right)$ be the sequence of Jordan curves in $\mathcal{F}$ and $a_{\infty}$ the arc of the limit curve $c_{\infty}$ provided by Proposition 3.1. Let $p, q$ denote the endpoints of $a_{\infty}$. Let $\varepsilon>0$ be very small with respect to $d(p, q)$, and let $r, \eta$ be as in Lemma 5.12. Note that $r \leq \eta \leq \varepsilon$. By construction, for $n$ large enough, there exist disjoint $\operatorname{arcs} a_{n}$ and $a_{n}^{\prime}$ of $c_{n}$ that lie in the $r$-neighbourhood of $a_{\infty}$ and meet both spheres $S(p, r)$ and $S(q, r)$.

Cutting out both ends of $a_{n}$ and $a_{n}^{\prime}$ if necessary, we may moreover assume that $a_{n}\left(\right.$ resp. $\left.a_{n}^{\prime}\right)$ has an endpoint $x_{n}\left(\right.$ resp. $\left.x_{n}^{\prime}\right)$ on the sphere $S(p, r)$, an endpoint $y_{n}$ (resp. $y_{n}^{\prime}$ ) on the sphere $S(q, r)$, and is otherwise drawn in $\mathbb{S}^{2} \backslash \overline{D(p, r)} \cup \overline{D(q, r)}$. Choose an arc $\alpha_{n, p}$ on $S(p, r)$ joining $x_{n}$ and $x_{n}^{\prime}$, and an $\operatorname{arc} \alpha_{n, q}$ on $S(q, r)$ joining $y_{n}$ and $y_{n}^{\prime}$. Then the union $\Gamma_{n}:=a_{n} \cup a_{n}^{\prime} \cup \alpha_{n, p} \cup \alpha_{n, q}$ is a Jordan curve which lies in the $r$-neighbourhood of $a_{\infty}$. It thus follows from Lemma 5.12 that the bounded component $B_{n}$ of $\mathbb{C} \backslash \Gamma_{n}$ lies in the $\eta$-neighbourhood of $a_{\infty}$.

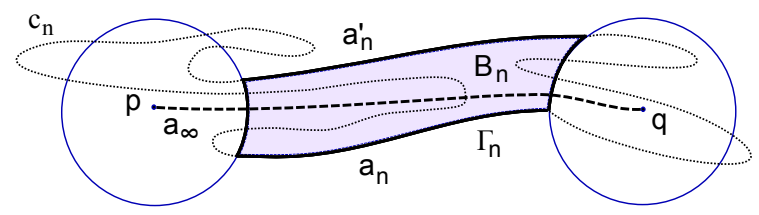

FiguRE 7 . The $\operatorname{arcs} a_{n}$ and $a_{n}^{\prime}$ on the Jordan curves $c_{n}$ and $\Gamma_{n}$, and the box $B_{n}$.

Observe that some $\operatorname{arcs}$ of $c_{n}$ may enter the box $B_{n}$. We thus introduce the connected component $U_{n}$ of $B_{n} \backslash c_{n}$ whose closure $\overline{U_{n}}$ contains the arc $a_{n}$. We claim that such a connected component $U_{n}$ does exist. This fact is easy when the quadrilateral $B_{n}$ is a rectangle, and the general case follows since Jordan's theorem provides us with a homeomorphism between $\overline{B_{n}}$ and a rectangle. Note that, since $\mathbb{S}^{2} \backslash U_{n}$ is connected, the domain $U_{n}$ is simply connected. A glance at Figure 7 may be useful, but keep in mind that the closure $\overline{U_{n}}$ does not always contain the arc $a_{n}^{\prime}$.

Choose two points $p_{n}$ and $q_{n}$ in $U_{n}$ such that $d\left(p, p_{n}\right)=r+\varepsilon$ and $d\left(q, q_{n}\right)=r+\varepsilon$. Then, Proposition 5.7 provides us with a pearl necklace $\mathcal{N}_{n}=\left(D_{n}(i) \mid i \in I_{n}\right)$ in the simply connected domain $U_{n}$, joining $p_{n}$ to $q_{n}$. Since $U_{n} \subset B_{n}$, Lemma 5.12 ensures that the diameters of all the disks that constitute this necklace are at most $\varepsilon$.

We are not done yet, since $\mathcal{N}_{n}$ is only a necklace in the domain $U_{n}$. Indeed the contact points of the disks $D_{n}(i)$ with the boundary $\partial U_{n}$, which occur in condition (3) of Definition 3.2. may either lie in $c_{n}$ (which is good) or on one of the arcs $\alpha_{n, p}$ or $\alpha_{n, q}$. Hence we choose a subnecklace $N_{n} \subset \mathcal{N}_{n}$, whose first disk meets the sphere $S(p, r+2 \varepsilon)$ and whose last disk meets the sphere $S(q, r+2 \varepsilon)$, and that is minimal with respect to these properties. This necklace $N_{n}$ is also a necklace in $\mathbb{S}^{2} \backslash c_{n}$. This necklace is $\varepsilon /(d(p, q)-4 \varepsilon)$-thin as required. 


\section{Finite Sequences of ReAl numbers}

We prove here an elementary fact on finite sequences of real numbers.

Proof of Proposition 3.4. Assume that there are no wells. This means that there exists $h_{2}>0$ such that, for any sequence $x=(x(i))_{i \in I_{x}} \in \mathcal{S}$ and $i<j<k$, one has either $x(i) \leq x(j)+h_{2}$ or $x(k) \leq x(j)+h_{2}$.

Assume that there are no pipes either. Choose $h_{1}=2 h_{2}$. Then, there exists a length $\ell$ such that any subsequence $(x(i))_{i \in J} \subset(x(i))_{i \in I_{x}}$ - where $x \in \mathcal{S}$ and $J \subset I_{x}$ is a subinterval of length at least $\ell$ - has an oscillation greater than $2 h_{2}$ : there exist $i, j \in J$ with $|x(i)-x(j)| \geq 2 h_{2}$.

We will prove that each sequence in $\mathcal{S}$ is a slide, with height $h_{3}:=h_{2}$ and slope $\sigma:=\frac{h_{2}}{\ell}$. Thus let $x=(x(i))_{i \in I} \in \mathcal{S}$ and choose $i_{0}$ such that $x\left(i_{0}\right)=\max _{i \in I_{x}} x(i)$. Let us work for example in the future of $i_{0}$.

Assume that $\left[i_{0}, i_{0}+\ell\right] \subset I_{x}$. Since there are no pipes and all $x(i)$ are bounded by $x\left(i_{0}\right)$, there exists $i_{1} \in\left[i_{0}, i_{0}+\ell\right]$ such that $x\left(i_{1}\right) \leq x\left(i_{0}\right)-2 h_{2}$. Since there are no wells, this implies that $x(i) \leq x\left(i_{0}\right)-h_{2}$ for any $i \in I_{x}$ with $i \geq i_{1}$.

Assume that $\left[i_{1}, i_{1}+\ell\right] \subset I_{x}$. Denying again the existence of pipes and wells yields an $i_{2} \in\left[i_{1}, i_{1}+\ell\right]$ with $x\left(i_{2}\right) \leq x\left(i_{0}\right)-3 h_{2}$, and ensures that $x(i) \leq x\left(i_{0}\right)-2 h_{2}$ for $i \geq i_{2}$. We go on and, as long as $\left[i_{k-1}, i_{k-1}+\ell\right] \subset I_{x}$, we get an integer $i_{k}$ in $\left[i_{k-1}, i_{k-1}+\ell\right]$ such that

$$
x(i) \leq x\left(i_{0}\right)-k h_{2} \quad \text { for any } i \in I_{x} \text { with } i \geq i_{k} .
$$

For all $i \geq i_{0}$ in $I_{x}$, one can choose $k$ such that $i \in\left[i_{k}, i_{k}+\ell\right]$. Note that, by construction, $i_{k} \leq i_{0}+k \ell$. Hence this $k$ is larger than $\frac{i-i_{0}-\ell}{\ell}$, and one has as required

$$
x(i) \leq x\left(i_{0}\right)-\frac{i-i_{0}-\ell}{\ell} h_{2} \leq x\left(i_{0}\right)+h_{2}-\frac{h_{2}}{\ell}\left|i-i_{0}\right| .
$$

\section{Pipes, Wells And Slides}

We put together the results of Sections 5.4 and 6 to finally prove Proposition 3.5, and hence Theorem 1.1.

Proof of Proposition 3.5. Proposition 3.3 provides us, for all $\varepsilon>0$, with a Jordan curve $c_{\varepsilon} \in \mathcal{F}$ and an $\varepsilon$-thin necklace $N_{\varepsilon}=\left(D_{\varepsilon}(i) \mid i \in I_{\varepsilon}\right)$ in $\mathbb{S}^{2} \backslash c_{\varepsilon}$. We let $\mathcal{S}_{\mathcal{F}}$ denote the family of sequences $x_{\varepsilon}=\left(x_{\varepsilon}(i) \mid i \in I_{\varepsilon}\right)$ associated to the necklaces $N_{\varepsilon}$, with $x_{\varepsilon}(i)=\log \operatorname{diam} D_{\varepsilon}(i)$ and apply Proposition 3.4 to $\mathcal{S}_{\mathcal{F}}$.

$\triangleright$ Suppose that there exist pipes in $\mathcal{S}_{\mathcal{F}}$. Shortening and shifting the intervals $I_{\varepsilon}$, we may extract from the family $\left\{c_{\varepsilon}, \varepsilon>0\right\}$ a sequence of Jordan curves $\left(c_{n}\right)_{n \in \mathbb{N}}$ and corresponding necklaces $N_{n}=\left(D_{n}(i)|| i \mid \leq n\right)$ such that the ratios

$$
\operatorname{diam} D_{n}(i) / \operatorname{diam} D_{n}(j) \quad(\text { for } n \in \mathbb{N} \text { and }|i|,|j| \leq n)
$$

of the diameters of these disks are uniformly bounded between $1 / \delta$ and $\delta$ for some $\delta>1$.

Applying a suitable conformal transformation of $\mathbb{S}^{2}$ that fixes $\infty$, we furthermore assume that the middle disk $D_{n}(0)$ of each necklace is always the unit disk $D(0,1) \subset \mathbb{C}$. Together with condition (1), this implies that, for a fixed $i \in \mathbb{Z}$, all the disks $D_{n}(i)$ (where $n \geq|i|$ ) live in a compact set of disks of the complex plane. Using a diagonal argument we may thus assume that, for each $i \in \mathbb{Z}$, the 
sequence $\left(D_{n}(i)\right)_{n \geq|i|}$ converges to a disk $D_{\infty}(i)$ with center $\omega_{i}$ and diameter between $1 / \delta$ and $\delta$. Going again to a subsequence, we may also assume that the sequence $\left(c_{n}\right)_{n \in \mathbb{N}}$ converges to $c_{\infty} \in \mathcal{K}$ when $n \rightarrow \infty$. The bounds on the diameters of the $D_{n}(i)$ 's and condition (2) force $\left|\omega_{i}\right| \rightarrow \infty$ when $|i| \rightarrow \infty$, so that the broken line $L=\bigcup_{i \in \mathbb{Z}}\left[\omega_{i}, \omega_{i+1}\right] \subset \mathbb{C}$ yields a proper embedding of $\mathbb{R}$ into $\mathbb{C}$. By Jordan's theorem, $\mathbb{C} \backslash L$ has two connected components. As a consequence of condition (3), the limit curve $c_{\infty}$ visits both connected components of $\mathbb{C} \backslash L$. This ensures that the limit $c_{\infty}$ is not a singleton, hence is a Jordan curve. However since, for all $n$, $c_{n}$ avoids the open set $\bigcup_{i} D_{n}(i)$, the limit curve $c_{\infty}$ does not meet $L$ and hence cannot be a Jordan curve (see the first drawing in Figure 8), a contradiction.

$\triangleright$ Assume now that there are wells in $\mathcal{S}_{\mathcal{F}}$. We proceed as in the previous case. This time, we shift the intervals so that the bottom of each well occurs for the index 0 , and apply a conformal normalisation so that the corresponding disk $D_{n}(0)$ is always the unit disk $D(0,1)$.

We obtain this time a sequence of Jordan curves $c_{n} \in \mathcal{F}$, a nondecreasing sequence of finite intervals $I_{n}$ that contains 0 , and a sequence of necklaces $N_{n}=$ $\left(D_{n}(i) \mid i \in I_{n}\right)$ in $\mathbb{S}^{2} \backslash c_{n}$ such that $D_{n}(0)=D(0,1)$, and such that the logarithms of the diameters of these disks, $x_{n}(i):=\log \operatorname{diam} D_{n}(i)$, satisfy

$$
x_{n}(0)=0, x_{n}(i) \geq 0 \text { for } i \text { in } I_{n} \text {, and } x_{n}(i) \geq n \text { for both endpoints } i \in I_{n} .
$$

Let $I \subset \bigcup_{n} I_{n}$ be the maximal subinterval containing 0 such that the sequence $n \mapsto x_{n}(i)$ is bounded when $i$ is an interior point of $I$. Note that this interval may be finite or infinite. By construction for any endpoint $i$ of $I$ the sequence $n \mapsto x_{n}(i)$ is unbounded. After going through a diagonal process, the sequence of disks $D_{n}(i)$ converges, when $n \rightarrow \infty$, to a disk $D_{\infty}(i)$ when $i$ is an interior point of $I$ and to a half-plane $D_{\infty}(i)$ when $i$ is an endpoint of $I$.

The conclusion follows as in the previous case: the limit $c_{\infty}=\lim _{n \rightarrow \infty} c_{n}$ avoids the shaded area and visits both components of its complementary set and hence cannot be a Jordan curve (see the second and third drawings in Figure 8), a contradiction.
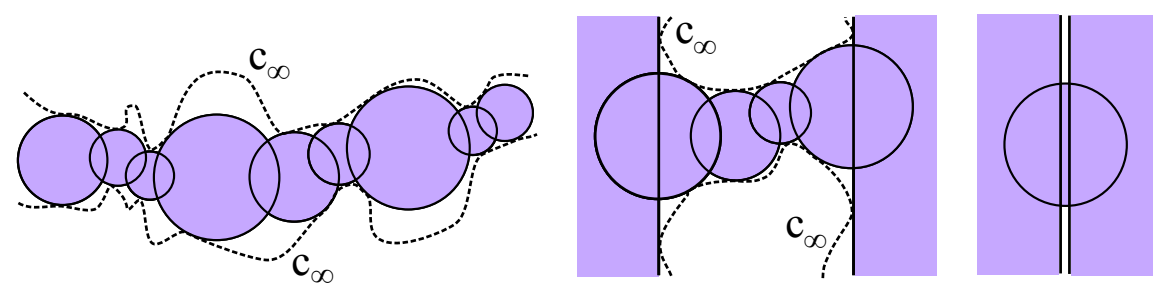

FiguRE 8. The limit curve and the limit of necklaces when respectively $I=\mathbb{Z}, \quad I$ is finite, and $I=\{-1,0,1\}$ in which case $\partial D_{\infty}(-1)=\partial D_{\infty}(1)$.

$\triangleright$ Finally, if all sequences in $\mathcal{S}_{\mathcal{F}}$ were slides with height $h_{3} \geq 0$ and slope $\sigma>0$, the ratio inverse of the thickness would be uniformly bounded,

$$
\operatorname{diam}\left(\bigcup_{i \in I_{\varepsilon}} D_{\varepsilon}(i)\right) / \max _{i \in I_{\varepsilon}} \operatorname{diam}\left(D_{\varepsilon}(i)\right) \leq 2 \sum_{i=0}^{\infty} e^{h_{3}} e^{-\sigma i} \leq 2 e^{h_{3}} /\left(1-e^{-\sigma}\right),
$$

a contradiction.

This also ends the proof of Theorem 1.1 


\section{Cantor Sets And the CONFormal group}

In this section we prove an analog of Theorem 1.1 where Jordan curves are replaced by Cantor sets.

8.1. Quasi-middle-third Cantor sets. Recall that a nonempty compact set $C$ is called a Cantor set if it is perfect and totally disconnected. The main example is the middle-third Cantor set

$$
C_{0}:=\left\{\sum_{n \geq 1} a_{n} 3^{-n} \mid a_{n}=0 \text { or } 2\right\} \subset[0,1] .
$$

Let $K \geq 1$. We will say that a Cantor set $C \subset \mathbb{S}^{2}$ is a $K$-quasi-middle-third Cantor set if $C$ is the image $C=f\left(C_{0}\right)$ of the middle-third Cantor set $C_{0} \subset \mathbb{S}^{2}$ under a $K$-quasiconformal homeomorphism $f: \mathbb{S}^{2} \rightarrow \mathbb{S}^{2}$.

The following theorem is an analog of Theorem 1.1

Theorem 8.1. Let $K \geq 1$. The set of all $K$-quasi-middle-third Cantor sets of $\mathbb{S}^{2}$ is a closed $G$-invariant subset of $\mathcal{K}_{0}$.

Conversely, any closed $G$-invariant subset of $\mathcal{K}_{0}$ which consists only of Cantor sets is included in the set of $K$-quasi-middle-third Cantor sets for some $K \geq 1$.

Corollary 8.2. A Cantor set $C \subset \mathbb{S}^{2}$ is a quasi-middle-third Cantor set if and only if its orbit closure $\overline{G C}$ in $\mathcal{K}$ consists only of points and Cantor sets.

We will just sketch the proof of Theorem 8.1 which is much shorter than the proof of Theorem 1.1. It follows from Lemmas 8.5, 8.6 and 8.7, combined with MacManus' condition described below.

8.2. MacManus' condition. Here is an analog of Ahlfors's arc condition (Theorem 2.4 for Cantor sets. We recall that $d$ denotes the canonical Riemannian metric on $\mathbb{S}^{2}$ and we denote by $B(x, r)$ the balls and by $\operatorname{diam}_{d}$ the diameter with respect to this metric.

Definition 8.3. Let $A>1$. A compact set $C \subset \mathbb{S}^{2}$ is $A$-uniformly perfect if, for all $x$ in $C$ and all $0<r<\operatorname{diam}_{d}(C)$, one has

$$
B(x, r) \cap C \not \subset B(x, r / A) .
$$

A compact set $C \subset \mathbb{S}^{2}$ is $A$-uniformly disconnected if, for all $x$ in $C$ and all $r>0$, the connected component of $x$ in the $r / A$-neighbourhood of $C$ is included in $B(x, r)$.

Theorem 8.4 (MacManus; see [14, Theorem 3]). A compact subset $C \subset \mathbb{S}^{2}$ is a quasi-middle-third Cantor set if and only if it is uniformly perfect and uniformly disconnected. The implied constants depend only on each other.

Let us also mention two related results: another characterization of quasi-middlethird Cantor sets (see [4, Corollary 2.1]), and a similar characterization of all compact metric spaces that are quasisymmetric to the middle-third Cantor set (see [6. Chapter 15]).

8.3. Limits of Cantor sets. The direct implication in Theorem 8.1 is a special case of the following analog of Proposition 2.2

Lemma 8.5 (Limits of quasi-middle-third Cantor sets). Let $\left(C_{n}\right)_{n \geq 1}$ be a sequence of $K$-middle-third Cantor sets in $\mathbb{S}^{2}$ that converges in $\mathcal{K}$ to a compact set $C_{\infty} \subset \mathbb{S}^{2}$ 
which is not a point. After going to a subsequence if necessary, there exist $K$ quasiconformal homeomorphisms $f_{n}: \mathbb{S}^{2} \rightarrow \mathbb{S}^{2}$ and $f_{\infty}: \mathbb{S}^{2} \rightarrow \mathbb{S}^{2}$ with $C_{n}=f_{n}\left(C_{0}\right)$, $C_{\infty}=f_{\infty}\left(C_{0}\right)$, and such that $f_{n} \rightarrow f_{\infty}$ uniformly on $\mathbb{S}^{2}$.

Proof. The proof is the same as for Proposition 2.2 using the "uniformly perfect" part of MacManus' condition to know that the limit $C_{\infty}$ contains at least three points.

The converse implication in Theorem 8.1 follows from MacManus' condition and the following two lemmas.

Lemma 8.6 (Nonuniformly perfect sequences of compact sets). Let $\left(C_{n}\right)_{n \geq 1}$ be a sequence of compact subsets of $\mathbb{S}^{2}$ such that $C_{n}$ is not $n$-uniformly perfect. After going to a subsequence if necessary, there exist elements $\gamma_{n} \in G$ such that $\left(\gamma_{n} C_{n}\right)$ converges in $\mathcal{K}$ to a nonperfect set $C_{\infty}$ containing at least two points.

Proof. By assumption, there exist $x_{n}$ in $C_{n}$ and $0<r_{n}<\operatorname{diam}\left(C_{n}\right)$ such that $B\left(x_{n}, r_{n}\right) \cap C \subset B\left(x_{n}, r_{n} / n\right)$. Fix $x_{0}$ in $\mathbb{S}^{2}$ and choose $\gamma_{n} \in G$ such that $\gamma_{n} x_{n}=x_{0}$ and $\gamma_{n}\left(B\left(x_{n}, r_{n}\right)\right)=B\left(x_{0}, 1\right)$. Going to a subsequence, $\left(\gamma_{n} C_{n}\right)$ converges to a compact set $C_{\infty}$ of $\mathbb{S}^{2}$ which contains $x_{0}$ as the only point in $B\left(x_{0}, 1\right)$, and is not a singleton. Hence the set $C_{\infty}$ is not perfect.

The second lemma is very similar to the first one.

Lemma 8.7 (Nonuniformly disconnected sequences of compact sets). Let $\left(C_{n}\right)_{n \geq 1}$ be a sequence of compact subsets of $\mathbb{S}^{2}$ such that $C_{n}$ is not n-uniformly disconnected. After going to a subsequence if necessary, there exist elements $\gamma_{n} \in G$ such that $\left(\gamma_{n} C_{n}\right)$ converges in $\mathcal{K}$ to a not totally disconnected set $C_{\infty}$.

Proof. The argument is also very similar. By assumption, there exist $x_{n}$ in $C_{n}$, $0<r_{n}<\pi$ and a finite subset $F_{n} \subset C_{n}$ containing $x_{n}$ such that the $r_{n} / n$ neighbourhood of $F_{n}$ is connected and meets the sphere $S\left(x_{n}, r_{n}\right)$. Fix $x_{0}$ in $\mathbb{S}^{2}$ and choose $\gamma_{n} \in G$ such that $\gamma_{n} x_{n}=x_{0}$ and $\gamma_{n}\left(B\left(x_{n}, r_{n}\right)\right)=B\left(x_{0}, 1\right)$. Going to a subsequence, $\left(\gamma_{n} C_{n}\right)$ converges to a compact set $C_{\infty}$ of $\mathbb{S}^{2}$ and $\left(\gamma_{n} F_{n}\right)$ converges to a compact set $F_{\infty} \subset C_{\infty}$ which contains $x_{0}$, is connected and meets the sphere $S\left(x_{0}, 1\right)$. Hence the set $C_{\infty}$ is not totally disconnected.

This ends the proof of Theorem 8.1 .

\section{ThuRston's LAMINATION AND MONOTONOUS PATHS}

We give in this last section an elementary and self-contained proof of the existence of monotonous paths (Proposition [5.6).

Throughout this section, $U \subset \mathbb{S}^{2}$ will denote a simply connected domain that avoids at least two points. We keep the notation of Sections 5.1 and 5.2

9.1. Thurston's lamination. Thurston's lamination is the lamination $\Lambda$ of $U$ by the arc of circles equal to the connected component of $\lambda\left(D_{p}\right)$ for some $p \in U$ (see Lemma 5.4).

Definition 9.1. Let $A$ be an $\operatorname{arc}$ of the lamination $\Lambda$. Let $a \in A$ be a point on this arc. A transverse $\tau$ to $(A, a)$ is a nontrivial segment on a geodesic of $D_{p}$ that meets $A$ orthogonally at the point $a$ and that admits this point $a$ as an endpoint. 
The following proposition will be useful for the construction of monotonous paths.

Proposition 9.2. Let $A \in \Lambda$ be an arc of the lamination, let $\Omega$ be one of the connected components of $U \backslash A$ and let $a_{1}, a_{2} \in A$ be two points on this arc. Then, there exist:

- Transverses $\tau_{i}$ to $\left(A, a_{i}\right)$ with second endpoints $x_{i}$ in $\Omega$ (for $i=1,2$ ) with $C_{x_{1}}=C_{x_{2}}$.

- A path $\gamma \subset C_{x_{1}}$ from $x_{1}$ to $x_{2}$ satisfying the following property. Let $\mathcal{B} \subset \Omega$ be the closed region bounded by the segments $\left[a_{1}, a_{2}\right] \subset A$, the transverses $\tau_{1}$ and $\tau_{2}$ and the path $\gamma$. Then each stratum $C_{m}$ that intersects $\mathcal{B}$ also intersects both $\tau_{1}$ and $\tau_{2}$.

The closed region $\mathcal{B}$ is called a well-combed box for $\left(\left[a_{1}, a_{2}\right], \Omega\right)$.

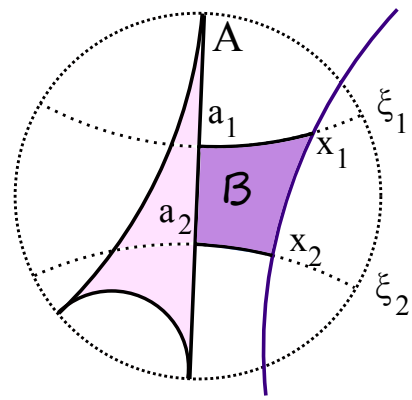

Figure 9. A box.

We begin with a lemma.

Lemma 9.3. Let $A$ be an arc of the lamination $\Lambda, a \in A$, and let $\tau$ be a transverse to $(A, a)$. Assume that $C_{a} \cap \tau=\{a\}$.

Let $p, q$ denote the endpoints of $A$. Let $\varepsilon>0$. Then, when $x \in \tau$ is close to a, the set of contact points $\partial D_{x} \cap \partial U$ meets both balls $B(p, \varepsilon)$ and $B(q, \varepsilon)$ and lies in their union.

In particular, if $\left(a_{n}\right)$ is a sequence of points on the transverse $\tau$ that converges to $a$, then the sequence of convex hulls $\left(C_{a_{n}}\right)$ converges to $A$.

Note that when $C_{a} \cap \Omega \neq \emptyset$ and the transverse $\tau$ is short enough, then $\tau$ lies in $C_{a}$. In this case, $C_{x}=C_{a}$ for any $x \in \tau$.

Proof. Let $p, q \in \partial D_{a} \cap \partial U$ denote the endpoints of the arc $A$. Let $\left(a_{n}\right)$ be a sequence of points of $\tau$ that converges to $a$.

To begin with, we assume that each point $a_{n}$ belongs to an $\operatorname{arc} A_{n}$ of the lamination $\Lambda$. Both endpoints of $A_{n}$ lie in $\partial U$; thus the arc $A_{n}$ intersects the boundary $\partial D_{a}$ in two points $p_{n}$ and $q_{n}$. Going to a subsequence, we may assume that $\left(p_{n}\right)$ and $\left(q_{n}\right)$ respectively converge to $\tilde{p} \in \partial D_{a}$ and $\tilde{q} \in \partial D_{a}$. We claim that $\{p, q\}=\{\tilde{p}, \tilde{q}\}$.

Indeed, the sequence of disks $\left(D_{a_{n}}\right)$ converges to $D_{a}$ (see Proposition [5.2); thus

$$
\operatorname{conv}_{D_{a_{n}}}\left(p_{n}, q_{n}\right) \longrightarrow \operatorname{conv}_{D_{a}}(\tilde{p}, \tilde{q}) \text {. }
$$



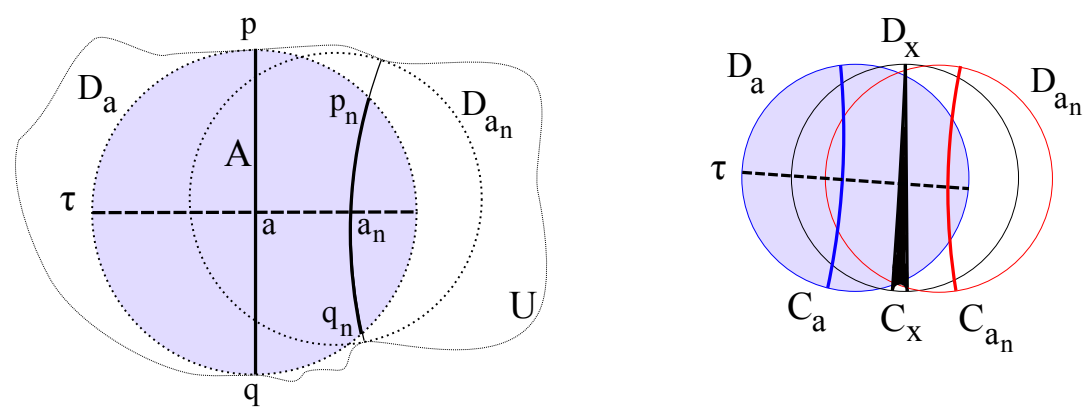

FiguRE 10. Transverses.

Since the points $a_{n}$ belong to $\operatorname{conv}_{D_{a_{n}}}\left(p_{n}, q_{n}\right)$ and since the point $a$ belongs to $\operatorname{conv}_{D_{a}}(p, q)$ while, by construction, the points $p_{n}$ and $q_{n}$ belong to the same connected component of $\partial D_{a} \backslash\{p, q\}$, we infer that $\{p, q\}=\{\tilde{p}, \tilde{q}\}$.

We now turn to the general case. Note that we can find a sequence $\alpha_{n} \in \tau$ such that $\alpha_{n} \neq a,\left(\alpha_{n}\right)$ converges to $a$ and $\alpha_{n}$ belongs to an arc $A_{\alpha_{n}}$ of the lamination $\Lambda$. For $x$ sitting in the segment $] a, \alpha_{n}\left[\subset \tau\right.$, the portion $C_{x} \cap D_{a}$ is included in the region delimited in $D_{a}$ by the $\operatorname{arcs} A$ and $A_{\alpha_{n}}$. When $n$ is large, we just proved that $A$ and $A_{\alpha_{n}}$ are close. The result follows as above, arguing that the disk $D_{x}$ depends continuously on $x$.

Proof of Proposition 9.2. Note that we have $D_{a_{1}}=D_{a_{2}}$ and $C_{a_{1}}=C_{a_{2}}$. The result is obvious when $A \neq C_{a_{1}}$ and $\Omega$ is the connected component of $U \backslash A$ that intersects $C_{a_{1}}$ since, in this case, we may find a box $\mathcal{B}$ that lies in $C_{a_{1}}$.

Assume now that $\Omega$ does not intersect $C_{a_{1}}$, and let $\left[a_{1}, \xi_{1}\right.$ [ and $\left[a_{2}, \xi_{2}\right.$ [ respectively denote the geodesic rays of $D_{a_{1}}$ that are orthogonal to $A$ at the points $a_{1}$ (resp. $a_{2}$ ) and point towards $\Omega$ (see Figure 9).

Lemma 9.3 provides a point $\left.x_{1} \in\right] a_{1}, \xi_{1}\left[\right.$ such that all the $C_{x}$ 's, where $x \in$ $\left[a_{1}, x_{1}\right] \subset \tau_{1}$, are close enough to $A$ so that they intersect $] a_{2}, \xi_{2}[$. We may even assume that $C_{x_{1}}$ is an arc and let $x_{2}:=C_{x_{1}} \cap T_{2}$. If $x_{1}$ is close to $a_{1}$, then $x_{2}$ is close to $a_{2}$ and all the $C_{y}$ 's - where $y \in\left[a_{2}, x_{2}\right] \subset \tau_{2}$ - do intersect $\tau_{1}$. We may then choose $\tau_{1}=\left[a_{1}, x_{1}\right]$ and $\tau_{2}=\left[a_{2}, x_{2}\right]$.

9.2. Piecewise monotonous paths. Recall that a path $\gamma:[0,1] \rightarrow U$ is monotonous if, for any parameters $0 \leq r \leq s \leq t \leq 1$, the point $\gamma(s)$ lies between $\gamma(r)$ and $\gamma(t)$ (see Definition 5.5). To strengthen our intuition, we first observe the following.

Lemma 9.4. A monotonous path $\gamma:[0,1] \rightarrow U$ with endpoints $x$ and $z$ crosses a convex hull $C_{y}$ if and only if $y$ lies between $x$ and $z$.

Proof. By definition of monotony, we know that $\gamma(t)$ lies between $x$ and $z$ for any $0 \leq t \leq 1$. Conversely, let $y$ be a point of $U$ that lies between $x$ and $z$ and such that $C_{x} \neq C_{y}$ and $C_{y} \neq C_{z}$. This means that $x$ and $y$ belong to two different connected components of $U \backslash C_{y}$, hence the path $\gamma$, which goes from $x$ to $z$, must cross $C_{y}$.

Our aim in this section is to prove that $U$ is monotonous-path-connected (this is Proposition 5.6). To prove this result, we will use the following alternative definition of monotony. 
Proposition 9.5. A path $\gamma:[0,1] \rightarrow U$ is monotonous if and only if it cuts each $C_{p}$ only once, namely if, for any point $p \in U$, the set $\gamma^{-1}\left(C_{p}\right) \subset[0,1]$ is connected.

Proof. Let $\gamma$ be monotonous. We proceed by contradiction, and assume that there exist $r<s<t$ in $[0,1]$ such that both $x=\gamma(r)$ and $z=\gamma(t)$ lie in $C_{x}$, while $y=\gamma(s) \notin C_{x}$. Since $\gamma$ is monotonous, we know that $x$ and $z$ lie in two distinct connected components of $U \backslash C_{y}$. This is a contradiction since $C_{x} \subset U \backslash C_{y}$ is connected.

Assume that $\gamma:[0,1] \rightarrow U$ is not monotonous; we may assume with no loss of generality that both points $x=\gamma(0)$ and $z=\gamma(1)$ lie in the same connected component $\Omega$ of $U \backslash C_{y}$, where $y:=\gamma(1 / 2)$. Define

$$
\begin{array}{ll}
t_{1}=\inf \left\{t>0 \mid \gamma(t) \in C_{y}\right\}, & b_{1}=\gamma\left(t_{1}\right), \\
t_{2}=\sup \left\{t<1 \mid \gamma(t) \in C_{y}\right\}, & b_{2}=\gamma\left(t_{2}\right) .
\end{array}
$$

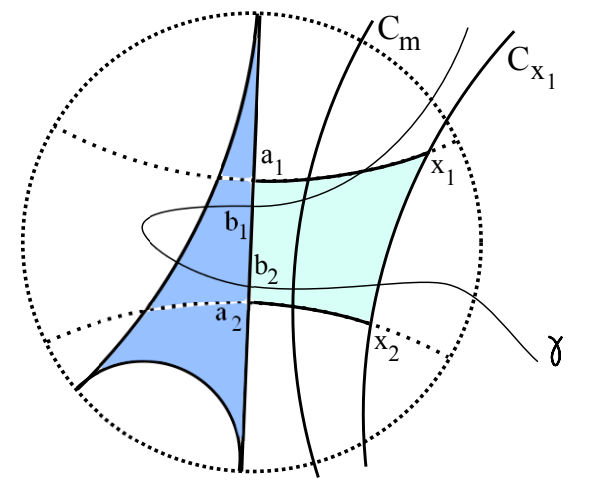

FiguRE 11. Equivalent definition of monotony.

It follows from the assumption and Lemma 5.4 that the points $b_{1}$ and $b_{2}$ belong to the same arc $A \subset \lambda\left(D_{y}\right)$ of the lamination $\Lambda$. Pick two points $a_{1}$ and $a_{2}$ on $A$ so that the segment $\left[b_{1}, b_{2}\right] \subset A$ lies in the open segment $] a_{1}, a_{2}[\subset A$. Proposition 9.2 provides a well-combed box $\mathcal{B}$ for $\left(\left[a_{1}, a_{2}\right], \Omega\right)$. Observe that any $C_{m}$ that meets the transverse $\tau_{1}$ at an interior point disconnects the box $\mathcal{B}$. Choosing $C_{m} \subset \Omega$ close enough to $A$, this ensures that there exist $\left.s_{1} \in\right] 0, t_{1}\left[\right.$ and $\left.s_{2} \in\right] t_{2}, 1[$ such that $\gamma\left(s_{1}\right)$ and $\gamma\left(s_{2}\right)$ both lie in $C_{m}$, hence that $\gamma^{-1}\left(C_{m}\right)$ is not connected.

Corollary 9.6. Let $A \in \Lambda$ be an arc of the lamination and $a \in A$. Let $\tau$ and $\tau^{\prime}$ be transverses to $(A, a)$ corresponding to each connected component of $U \backslash A$. If these transverses are short enough, their union $\tau \cup \tau^{\prime}$ is the image of a monotonous path.

Proof. Proposition 9.2 implies that both $\tau$ and $\tau^{\prime}$ are images of monotonous paths. The equivalent definition of monotony provided by Proposition 9.5 implies that their union $\tau \cup \tau^{\prime}$ is also the image of a monotonous path.

Lemma 9.7. The domain $U$ is locally monotonous-path-connected: each point $x \in$ $U$ admits arbitrarily small neighbourhoods $V$ such that any pair of points $y, z \in V$ may be joined by a monotonous path. 
Proof. Assume first that $x$ belongs to the interior of $C_{x}$. Then, we may take for $V$ any neighbourhood of $x$ included in $C_{x}$.

Suppose now that $x$ belongs to an arc $A$ of the lamination. Pick two points $a_{1}$ and $a_{2}$ on $A$ such that $x$ lies in the open segment $] a_{1}, a_{2}\left[\subset A\right.$. For the segment $\left[a_{1}, a_{2}\right]$, and each one of the connected components $\Omega$ of $U \backslash A$, Proposition 9.2 provides us with a well-combed box. The union of these two boxes is a neighbourhood $V$ of $x$. It can be made arbitrarily small by choosing the points $a_{i}$ close to $x$ and the transverses short. We claim that $V$ is monotonous-path-connected.

Let indeed $y, z \in V$. Choose paths $\gamma_{y} \subset C_{y} \cap V$ and $\gamma_{z} \subset C_{z} \cap V$ that respectively join the points $y$ and $z$ to points $y_{1}$ and $z_{1}$ that lie on one of the transverses to $\left(A, a_{1}\right)$ that bound the domain $V$. It follows from Corollary 9.6 that the concatenated path $\mu=\gamma_{z}^{-1} *\left[y_{1}, z_{1}\right] * \gamma_{y}$ is monotonous.

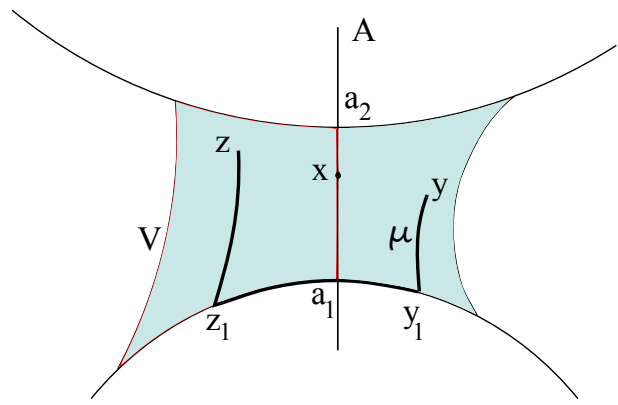

FiguRE 12. A neighbourhood of $x$ that is monotonous-path-connected.

Definition 9.8. A path $\gamma:[0,1] \rightarrow U$ is piecewise-monotonous if there exists an interval subdivision $0=t_{0}<t_{1} \cdots<t_{n}=1$ such that each restriction $\gamma_{\mid\left[t_{i}, t_{i+1}\right]}$ is a monotonous path for $i=0, \cdots, n-1$.

Corollary 9.9. The domain $U$ is piecewise-monotonous-path connected.

Proof. The proof is an immediate consequence of Lemma 9.7, since $U$ is connected.

\subsection{Monotonous paths.}

Proof of Proposition 5.6. We just proved that $U$ is piecewise-monotonous-path connected. The fact that $U$ is monotonous-path-connected will thus be an immediate consequence of the following proposition.

Proposition 9.10. Let $x, y$ and $z$ be three points in $U$. Assume that there exists a monotonous path $\gamma_{1}$ from $x$ to $y$ and a monotonous path $\gamma_{2}$ from $y$ to $z$. Then, there exists a monotonous path from $x$ to $z$.

Proof. Let both paths $\gamma_{i}(i=1,2)$ be parameterized by $[0,1]$.

(1) Assume first that $y$ lies inbetween $x$ and $z$. We claim that, in this case, the concatenated path $\gamma:=\gamma_{2} * \gamma_{1}$ is monotonous. Were it not the case, Proposition 9.5 would provide a point $p \in U$ (with $p \notin C_{y}$ ) such that $\gamma^{-1}\left(C_{p}\right)$ is not connected. Since both paths $\gamma_{1}$ and $\gamma_{2}$ are monotonous, this forces both $\gamma_{1}^{-1}\left(C_{p}\right)$ and $\gamma_{2}^{-1}\left(C_{p}\right)$ 

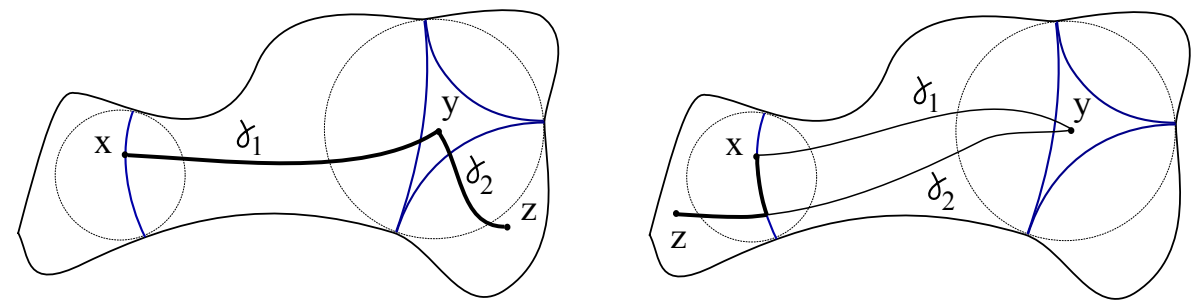

FiguRE 13. A piecewise-monotonous path yields a monotonous one $((1)$ and $(2))$.

to be nonempty. Hence the stratum $C_{p}$, which is connected, would intersect two distinct connected components of $U \backslash C_{y}$. This is a contradiction.

(2) We now assume that $x$ lies inbetween $y$ and $z$. Since the path $\gamma_{2}$ is monotonous, Lemma 9.4 ensures that there exists $t_{0} \in[0,1]$ with $\gamma_{2}\left(t_{0}\right) \in C_{x}$. Choose a path $\gamma_{3}:[0,1] \rightarrow C_{x}$ from $x$ to $\gamma_{2}\left(t_{0}\right)$. Then, the concatenated path $\left(\gamma_{2}\right)_{\mid\left[t_{0}, 1\right]} * \gamma_{3}$ joins $x$ to $z$ and is monotonous.

In case $z$ lies inbetween $x$ and $y$, the proof is similar.

(3) We now proceed with the last configuration, where none of the points $x, y, z$ lies inbetween the other two. We want to produce a monotonous path from $x$ to $z$.

As above, we will construct our monotonous path from $x$ to $z$ by cutting out a subpath of $\gamma_{2} * \gamma_{1}$, and replacing it by a shortcut that lies in a stratum. Lemma 9.4 ensures that

$$
J_{1}:=\left\{s \in[0,1] \mid \exists t \in[0,1] \text { with } C_{\gamma_{1}(s)}=C_{\gamma_{2}(t)}\right\}
$$

is a subinterval of $[0,1]$ containing 1 and that

$$
J_{2}:=\left\{t \in[0,1] \mid \exists s \in[0,1] \text { with } C_{\gamma_{1}(s)}=C_{\gamma_{2}(t)}\right\}
$$

is a subinterval containing 0 . Define $T_{1}=\inf J_{1}$ and $T_{2}=\sup J_{2}$.

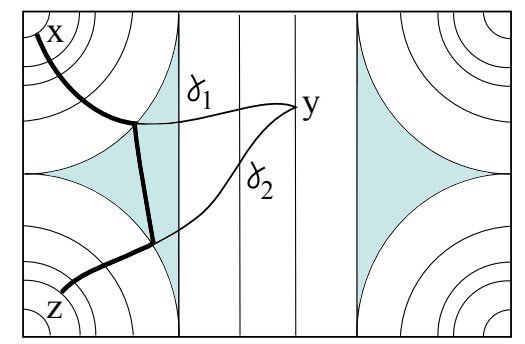

Figure 14. A piecewise-monotonous path yields a monotonous one (3).

Since the normal disk $D_{x}$ depends continuously on $x \in U$ (see Proposition 5.2), it follows that $D_{\gamma_{1}\left(T_{1}\right)}=D_{\gamma_{2}\left(T_{2}\right)}$, so that $C_{\gamma_{1}\left(T_{1}\right)}=C_{\gamma_{2}\left(T_{2}\right)}$. Now let $\gamma_{3}$ be a path drawn in the stratum $C_{\gamma_{1}\left(T_{1}\right)}$ and that goes from $\gamma_{1}\left(T_{1}\right)$ to $\gamma_{2}\left(T_{2}\right)$. The choice of $T_{1}$ and $T_{2}$ and Proposition 9.5 ensures that the path $\mu:=\gamma_{2 \mid\left[T_{2}, 1\right]} * \gamma_{3} * \gamma_{1 \mid\left[0, T_{1}\right]}$, that goes from $x$ to $z$, is monotonous. 


\section{REFERENCES}

[1] Lars V. Ahlfors, Quasiconformal reflections, Acta Math. 109 (1963), 291-301. MR.0154978

[2] Lars V. Ahlfors, Lectures on quasiconformal mappings, Manuscript prepared with the assistance of Clifford J. Earle, Jr., Van Nostrand Mathematical Studies, No. 10, D. Van Nostrand Co., Inc., Toronto, Ont.-New York-London, 1966. MR0200442

[3] Alexander M. Blokh, Robbert J. Fokkink, John C. Mayer, Lex G. Oversteegen, and E. D. Tymchatyn, Fixed point theorems for plane continua with applications, Mem. Amer. Math. Soc. 224 (2013), no. 1053, xiv+97pp., DOI 10.1090/S0065-9266-2012-00671-X. MR.3087640

[4] Petra Bonfert-Taylor and Edward C. Taylor, Quasiconformally homogeneous planar domains, Conform. Geom. Dyn. 12 (2008), 188-198, DOI 10.1090/S1088-4173-08-00189-6. MR2461511

[5] Martin Bridgeman and Richard D. Canary, The Thurston metric on hyperbolic domains and boundaries of convex hulls, Geom. Funct. Anal. 20 (2010), no. 6, 1317-1353, DOI 10.1007/s00039-010-0102-7. MR2738995

[6] Guy David and Stephen Semmes, Fractured fractals and broken dreams: Self-similar geometry through metric and measure, Oxford Lecture Series in Mathematics and its Applications, vol. 7, The Clarendon Press, Oxford University Press, New York, 1997. MR.1616732

[7] D. B. A. Epstein and A. Marden, Convex hulls in hyperbolic space, a theorem of Sullivan, and measured pleated surfaces [MR0903852], Fundamentals of hyperbolic geometry: selected expositions, London Math. Soc. Lecture Note Ser., vol. 328, Cambridge Univ. Press, Cambridge, 2006, pp. 117-266. MR2235711

[8] D. B. A. Epstein, A. Marden, and V. Markovic, Quasiconformal homeomorphisms and the convex hull boundary, Ann. of Math. (2) 159 (2004), no. 1, 305-336, DOI 10.4007/annals.2004.159.305. MR2052356

[9] Frederick W. Gehring and Kari Hag, The ubiquitous quasidisk, Mathematical Surveys and Monographs, vol. 184, American Mathematical Society, Providence, RI, 2012. With contributions by Ole Jacob Broch. MR2933660

[10] Henri Gillet and Peter B. Shalen, Dendrology of groups in low Q-ranks, J. Differential Geom. 32 (1990), no. 3, 605-712. MR 1078160

[11] Yoshinobu Kamishima and Ser P. Tan, Deformation spaces on geometric structures, Aspects of low-dimensional manifolds, Adv. Stud. Pure Math., vol. 20, Kinokuniya, Tokyo, 1992, pp. 263-299. MR.1208313

[12] Ravi S. Kulkarni and Ulrich Pinkall, A canonical metric for Möbius structures and its applications, Math. Z. 216 (1994), no. 1, 89-129, DOI 10.1007/BF02572311. MR1273468

[13] O. Lehto and K. I. Virtanen, Quasiconformal mappings in the plane, 2nd ed., Die Grundlehren der Mathematischen Wissenschaften, Band 126, Springer-Verlag, New York-Heidelberg, 1973. Translated from the German by K. W. Lucas. MR 0344463

[14] Paul MacManus, Catching sets with quasicircles, Rev. Mat. Iberoamericana 15 (1999), no. 2, 267-277, DOI 10.4171/RMI/256. MR1715408

[15] Lex G. Oversteegen and Edward D. Tymchatyn, Extending isotopies of planar continua, Ann. of Math. (2) 172 (2010), no. 3, 2105-2133, DOI 10.4007/annals.2010.172.2105. MR.2726106

[16] Ch. Pommerenke, Boundary behaviour of conformal maps, Grundlehren der Mathematischen Wissenschaften [Fundamental Principles of Mathematical Sciences], vol. 299, Springer-Verlag, Berlin, 1992. MR1217706

Département de Mathématiques, Université Paris-Sud, Orsay 91405, France

E-mail address: yves.benoist@math.u-psud.fr

Département de Mathématiques, Université Paris-Sud, Orsay 91405, France

E-mail address: dominique.hulin@math.u-psud.fr 